\title{
Boiling Two Phase Flow in Microchannels: a Review
}

\author{
Ahmed Jassim Shkarah"1,2*, Mohd. Yusoff Bin Sulaiman and Md. Razali Bin Hj Ayob \\ ${ }^{1}$ Faculty of Mechanical Engineering, Universiti Teknikal Malaysia Melaka (UTeM), Melaka, Malaysia; \\ ahmedshkarah@gmail.com; yusoff@utem.edu.my, razali@utem.edu.my \\ 2Department of Mechanical Engineering, Thi-Qar University, 64001 Nassiriya, Iraq
}

\begin{abstract}
The studies in boiling water in microchannel that are shaped along turbine blades has been examined since the 1970s as a means to dissipating large amounts of heat. Later, similar geometries could be found in cooling systems for computers, fusion reactors, rocket nozzles, avionics, hybrid vehicle power electronics, and space systems. This paper addresses: (a) dissipation high heat flux by two phase flow (b) how the geometry of micro channels affects their activity i.e. of circular and non-circular geometry, and (c) means to enhance this performance. It is shown that despite many hundreds of publications attempting to predict the performance of two-phase microchannel heat sinks, there are only a handful of predictive tools that can tackle broad ranges of geometrical and operating parameters or different fluids. There are a lot of complications for the development of these tools because of insufficient databases and also the drastic differences in boiling behavior of different fluids in microchannel. It is shown that cutting-edge heat transfer enhancement techniques, such as the use of nanofluids and carbon nanotube coatings, with proven merits to single-phase macrosystems, may not offer similar advantages to microchannel heat sinks. The boiling flow regimes in microchannels were categorized as the confined bubble, slug flow, and annular flow depending mainly on the length of the bubble. Based on the dominant flow regimes in microchannels, a steady slug-annular flow model is proposed to predict the pressure drop and heat transfer characteristics. As a correlation method, the homogeneous flow model with empirical correlations was applied to the boiling flow in microchannels. In addition, this review concludes that boiling flow in microchannel can be an effective way to eliminate heat from the heat sinks.
\end{abstract}

Keywords: Boiling, Heat transfer, Two-Phase Flow.

\section{Nomenclature}

Co' $=$ confinement number

$\mathrm{m}=$ overall surface efficiency

$\rho=$ density, $\mathrm{kg} / \mathrm{m} 3$

$\sigma=$ surface tension, $\mathrm{N} / \mathrm{m}$

$\mathrm{U}=$ flow velocity, $\mathrm{m} / \mathrm{s}$

$\mathrm{t}=$ residence time of a slug, $\mathrm{s}$

$\mathrm{T}=$ temperature, ${ }^{\circ} \mathrm{C}$

$\mathrm{R}^{\prime}=$ thermal resistance, $\mathrm{mK} / \mathrm{W}$

$\mathrm{m}=$ mass flow rate, $\mathrm{kg} / \mathrm{s}$

$\mathrm{N}=$ total number of device layers

$\mathrm{L}=$ length, $\mathrm{m}$

$\mathrm{La}=$ Laplace length scale (capillary length), $\mathrm{m}$

$\mathrm{k}=$ thermal conductivity, $\mathrm{W} / \mathrm{mK}$
$\mathrm{G}=$ mass flux, $\mathrm{kg} / \mathrm{m} 2 \mathrm{~s}$

$D_{h} \quad=$ hydraulic diameter, $\mathrm{m}$

$\mathrm{W}=$ width, $\mathrm{m}$

$\mathrm{w} \quad=$ channel pitch, $\mathrm{m}$

$\sigma=$ surface tension, $\mathrm{N} / \mathrm{m}$

$g \quad=$ gravitational acceleration, $\mathrm{m} / \mathrm{s} 2$

$S=$ suppression factor

$h=$ heat transfer coefficient, $\mathrm{W} / \mathrm{m} 2 \mathrm{~K}$

$f=$ friction factor

$-\phi_{l}^{2}=$ two-phase pressure drop multiplier

$X=$ Martinelli parameter

$C=$ constant

$E \quad=$ Enhancement factor

${ }^{*}$ Corresponding author:

Ahmed Jassim Shkarah (ahmedshkarah@gmail.com) 


$$
\begin{array}{ll}
\mathrm{K}= & \text { head loss coefficient } \\
\mathrm{i}= & \text { specific enthalpy, } / \mathrm{kg} \\
\mathrm{h}= & \text { heat transfer coefficient, W/m2K } \\
\mathrm{f}= & \text { friction factor }
\end{array}
$$

\section{Introduction}

The area of convective boiling flow and heat transfer in microchannels has been intensively investigated for the last decade. Rapid development of microfluidic devices has triggered the demand for a comprehensive understanding of mechanisms involved with heat transfer in boiling flow through micro-geometries [1-3]. This importance is reiterated by the increasing number of applications, in which phase change is used as the principal mechanism to conduct or remove heat. These fields include electronic circuit cooling, mini heat exchangers, bioengineering, aerospace, etc. $[4,5]$. Other areas of research include cooling of laser diode arrays, micro Joule-Thompson cooling devices, and the evaporator and condenser sections of micro vapor compression cycles [6]. In this review paper, we are going to review boiling flow phenomenon or flow boiling heat transfer phenomenon in two phase flow microchannel heat sinks.

\section{Two-phase Flow in Microchannel Heat Sinks}

In microchannel there are some thermal challenges, removing high heat flux in small area, reducing the pumping power and try to reach high thermal efficiency, to solve these thermal challenges, advanced cooling solutions are required. A reasonable amount of research has been focused on advanced microprocessor cooling over the years, with a significant number of advances made in the field of micro machined heat sink technology [7-12]. MicroChannel heat sinks have received much attention after Tuckerman and Pease demonstrated the removal of $790 \mathrm{~W} / \mathrm{cm}^{2}$ by using forced single-phase liquid convection $[13,14]$. Figure 1 shows the schematic of the microchannel heat sink fabricated on the back side of high power generation micro-processor. These heat sinks feature coolant flow through a series of parallel microchannels with cross-sectional dimensions ranging from 10 to 500 pm [15]. By removing the thermal interface material for attachment of the heat sink to the chip, this configuration significantly reduces the packaging level thermal resistance and hence leads to lowered junction temperatures.

$\begin{array}{ll}\mathrm{L}= & \text { liquid } \\ \mathrm{i}= & \text { core-film interface, layer index } \\ \mathrm{v}= & \text { vapor } \\ t_{p}= & \text { two-phase }\end{array}$

Two-phase microchannel heat sinks have emerged in recent years as a prime solution for thermal management of next generation high performance ICs. The combination of small channel size and flow boiling offers many advantages such as large thermal capacity and low thermal resistance between the chip and the coolant [10]. Furthermore, boiling convection requires less coolant flow rate and pumping power for a removing a given amount of heat in comparison to single-phase convection. In so doing, microchannel cooling can potentially provide improvements in temperature uniformity with hotspots, and occupy a very small volume at the chip level.

\section{Boiling Flow in Microchannel}

Modeling of transport phenomena in boiling flow is based on the understanding of fundamental mechanisms. The same fundamental mechanisms also in turn lead to empirical correlations. Conventional boiling heat transfer correlations take into account two mechanisms; nucleate boiling and forced convection [15]. Theories for assessing the relative contribution of these two separate mechanisms have been the subject of much debate, though several correlations have proven to yield satisfactory results over their range of applications. The pressure drop equations consist of acceleration and friction contributions which include empirical correlations for void fraction and friction factor.

Many experiments validated and improved these empirical correlations, which in some cases have shown good performance in limited applications [16]. However, their agreement with experimental data may be explained by the incorporation of empirical constants rather than being seen as confirmation of having a sound physical basis. It is therefore desirable to examine the flow regimes

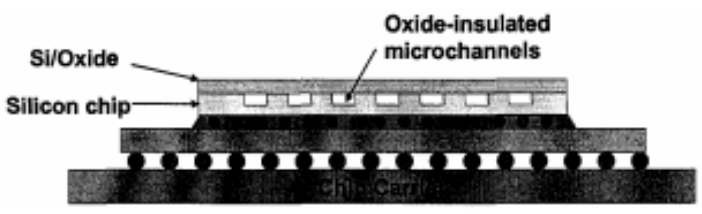

Figure 1. Schematic diagram of a microchannel heat sink fabricated on the backside of a chip. 
and transport mechanisms that occur during evaporation in a channel before deriving or selecting an appropriate correlation $[17,18]$. Following section explains the flow boiling heat transfer phenomenon in micro channels.

\section{Flow Boiling Heat Transfer in Microchannels}

The boiling flow in microchannels has been actively investigated due to the high heat transfer efficiency by the combination of boiling and small channel size. A recent progress on boiling flow transfer of heat in tubes $\mathrm{f}$ small diameter has been extensively reviewed by Nakayama and Yabe, Kew and Cornwell, Kandlikar, and Thome [19-21]. The review here presents trends found in recent studies and an assessment of what has been achieved so far. The conventional correlation as follows:

$$
h_{t p}=\left(h_{n b}^{n} S+h_{c v}^{n}\right)^{\frac{1}{n}}
$$

where, $\mathrm{n}$ is an arbitrary constant, generally considered to be unity. The nucleate boiling component $h_{n b}$ is a strong function of wall superheat and wall heat flux while the forced convection component is dependent on the mass flux $G$ and quality $x$. Chen argued that the high flow velocity suppresses nucleate boiling, and introduced suppression factor $S$ to be multiplied to the nucleate boiling term [22]. The convection term, which is independent of heat flux, is written as $h_{c v}=h_{i} E$. In choosing an empirical correlation, it is critical that the dominant heat transfer mechanism in the correlation should satisfy the physical basis of observed heat transfer characteristics and flow patterns $[22,23]$.

To explain the experimental data, they applied reasoning from macroscale boiling to concluded that saturated boiling heat transfer process is governed by nucleate boiling mechanism in microchannel. In contrast, others reported that the heat transfer is influenced by heat flux as well as mass flow rate and quality $[18,24]$.

As a microchannel heat sink receives a lot of attention, experiments have been performed with water as a working fluid [7]. Kandlikar reported that the heat transfer is a function of the mass flux and quality from their boiling experiments in narrow channels. He concluded that convective term is dominant, and recommended Kandliakar's correlation for high mass flux application [19]. Wu and Cheng performed experiments with a circular tube with a $2.98 \mathrm{~mm}$ diameter and found that the dominant heat transfer mechanism changes according to the wall superheat, such that nucleate boiling dominates at low superheat and convective boiling is dominant at high superheat [25].

$\mathrm{Qu}$ and Mudawar reported that the heat transfer coefficient in microchannels has a different behavior from that in macrochannels with respect to vapor quality. To explain their experimental data, they proposed correlations for liquid entrainment and deposition rate for an annular flow model [26]. Other authors proposed a new heat transfer model based on a slug flow and was able to successfully explain the reported behavior of the heat transfer coefficient $[23,24]$. Those models demonstrated that the major heat transfer mechanism in microchannels is not nucleate boiling but film boiling. The model employs an empirical correlation to predict the thickness of the liquid film followed by liquid slugs and three arbitrary variables to fit the experimental data [21]. The experimental correlations used in their models may have applicable limits and should be verified by other experiments.

\section{Macro- and Micro-Boiling Process}

Many theories, models and correlations have been developed for boiling flow in conventional size channels. As microscale boiling process has emerged as an important area of industrial application, the applicability of conventional models and correlations to small size channels requires further clarification $[17,21]$. In addition, there is necessity to establish a clear threshold where macroscale conditions give way to microscale boiling phenomena with regards to flow characteristics in a channel $[9,10,27]$.

For example, Kandlikar recommended the following classification depending on chronicle change of their application: microchannels (10-200 pm), minichannels (200 pm-3 mm), and conventional channels (A > $3 \mathrm{~mm})$ $[19,20]$. However, such transition criteria are somewhat arbitrary and their applicability to the internal boiling process is not certain. Since the phase-change phenomena in the microscale show a distinct difference from the macroscale, the criteria should be defined with respect to the influence of small channel size on the physical mechanisms of the boiling process. The criteria determine the applicability of conventional knowledge on internal boiling [2]. 
A more general threshold could be addressed in terms of the bubble departure diameter, and based on the fundamental boiling flow phenomena [28]. The distinct phenomenological characteristics are that the abrupt phase change occurs from liquid to boiling flow and the smallest size of an active bubble is comparable to the channel size. The smallest active bubble in a heated channel flow is the bubble departing from its incipient position [29]. As a bubble departs, it will generate a disturbance in the flow, increase heat transfer from the wall and grow or coerce by interacting with neighboring bubbles $[10,29,30]$.

One can potentially use the bubble departure diameter in nucleate pool boiling as the criterion. Note that this concept may not be applied to convective internal boiling. For an internal convection boiling process, Kew and Cornwell recommended a criterion of Co' $>0.5$ based on their experimental observation $[15,31]$. The confinement number $\mathrm{Co}$ is defined as:

$$
C_{o}^{\prime}=\left[\frac{\sigma}{g\left(\rho_{l}-\rho_{v}\right) D_{h}^{2}}\right]^{\frac{1}{2}}
$$

where, $\sigma$ the surface tension, $g$ is gravitational acceleration, and $D_{h}$ is the hydraulic diameter of the channel. The density is denoted by $\rho$ and subscripts I and v represents liquid and vapor, respectively. For water at atmospheric pressure, the threshold diameter given by this criterion is about $5 \mathrm{~mm}$. In new model authors proposed another criterion of $D_{h} / \mathrm{La}<0.3[11,13]$, where La is the Laplace length scale, also known as capillary length, defined as:

$$
L a=\left[\frac{\sigma}{g\left(\rho_{l}-\rho_{v}\right)}\right]^{\frac{1}{2}}
$$

The threshold diameter for water at atmospheric pressure is $750 \mathrm{pm}$ and decreases as the saturation pressure increases. These two criteria basically consider the balance between the surface tension and buoyancy forces [32]. They lack the influence of the flow conditions which should be accounted for and cannot provide the criterion to microscale transition in a convective boiling process $[8,12]$. Furthermore, it has been reported that the buoyancy force is suppressed in microchannels and can be considered negligible. While $\mathrm{Qu}$ and Mudawar develop a methodology of predicting the bubble departure and incipient heat flux in microchannels $[16,25,26]$.

\section{Numerical Modeling of Boiling Heat Transfer}

Lee and Lee and Warrier et al. proposed a correlation, assuming that forced convection is dominant. These two microchannel correlations yield different values to those obtained by conventional correlations. The correlation by Lee and Lee is developed for very narrow channels and its extrapolation beyond its limit may not accurate [23]. In addition, the Warrier correlation shows different behavior in comparison to others and is similar to that of the experimental data of $\mathrm{Qu}$ et al. However, the correlation by Warrier et al. is from the experiment with FC-84, whose thermo physical properties is similar to F-113, and may have limitation in its application to other fluids [25]. Further verification of the correlation is necessary.

$$
h_{t p}=E h_{1}
$$

The heat transfer mechanisms of microchannel boiling have not yet been fully understood. Still, no comparison has been made between independent data for the same conditions, e.g., channel size, fluid, flow rate, and heat flux. One of the unique characteristics of the microchannel boiling is that it experiences high pressure drop. Kandlikar [15] performed correlations and proposed following thing:

$$
h_{t p}=(S+E) h_{1}
$$

In analyzing the experimental data, the enthalpy change of the two-phase mixture due to the saturation pressure change should be accounted for in determining the local quality. Several previous studies analyzed the heat transfer data in microchannels by applying the macro-boiling logic to the microscale, which yields that nucleate boiling is dominant $[12,17]$. There are several phenomenological models proposed to simulate the boiling heat transfer in microchannels and to conclude that film boiling is dominant, but their experimental correlations are still in question [8]. There are issues on fluctuation and instability of boiling heat transfer in microchannels. Although they are not covered in this review, its importance in designing the microchannel device should not be neglected. The two numerical based approaches concluded by Chen and Shah $[16,22,32]$ respectively are as follows:

$$
\begin{aligned}
& h_{t p}=h_{n b} S+h_{1} E \\
& h_{t p}=\max (S+E) h_{1}
\end{aligned}
$$




\section{Experimental Modeling of Boiling Heat Transfer}

A large number of studies on fundamental theory and experiments have been conducted to characterize convective boiling flow in microchannels. The past work indicates that the boiling flows in microchannels show distinct behaviors, especially, in boiling transition, flow regimes and heat transfer characteristics [20]. Bowers and Mudawar uses the channel size ranged from $510 \mathrm{pm}$ to $2.54 \mathrm{~mm}$ and the refrigerant R-113 was used as a coolant to perform experiment. The experimental data are well correlated with the calculation by the homogeneous model with a $\pm 30 \%$ standard deviation [33].

Hetsroni et al. performed an experiment to investigate the boiling flow patterns in microchannels and reported a non-uniform temperature distribution [8]. Zhang et al. developed single-microchannel testing rigs with rectangular silicon/glass channels of 30-60 pm in hydraulic diameter and nearly-constant heat flux conditions. For the practical application of VLSI chip cooling using two-phase microchannels with water as a coolant, they developed a conjugate conduction- convection heat transfer model employing a homogeneous flow model for the prediction of boiling flow. Based on their boiling pattern observations, they proposed a compact annular flow model for boiling flow in microchannels. The simulation agrees reasonably well with experimental data while their annular flow model showed slight underpredictions of the wall temperature and pressure drop [24].

Jiang et al. investigated the thermal and hydraulic characteristics. He designed same concept of heat sink using a homogeneous two-phase model. The prediction using the homogeneous model agrees well with their experimental measurements of pressure drop and average chip temperature [8]. Qu et al. performed measurement and predictions of saturated flow boiling heat transfer and pressure drop in a water-cooled microchannel heat sink [26]. They also developed an annular flow model for the boiling flow in a rectangular microchannel. Although droplets were not experimentally observed, they assumed that liquid droplets are entrained at the front end of the boiling region and deposited to the liquid film. To correlate the simulation to experimental data, empirical correlations are proposed for the entrainment and deposition rates of liquid droplets [27].
Jacob and Thome proposed a slug flow model to predict the convective heat transfer coefficient of microchannel boiling flow. Due to the added complexity, their slug flow model requires the determination of four critical parameters for an accurate fit of the data, which are the liquid film thickness and others. Their predictions agree well with experimental data using refrigerants. Their model has demonstrated that the major heat transfer mechanism in microchannel boiling is film evaporation through a thin liquid film on the wall $[8,12]$.

\section{Pressure Effects on Flow Boiling Instabilities}

The prediction of pressure effects is critical in designing multiphase microchannel devices, since it is closely related to the pumping power consumption of the device. However, the accuracy of prediction is generally not very good and the standard deviation of $30 \%$ is typical even in the most advanced models [18]. There are two empirical models easily found in the literature: flow model of homogeneous nature and flow model separated nature. Each model will be presented here and then previous experimental findings are provided [11]. Generally, phenomenological models perform better than empirical correlations, at the expense of additional complexity, but are omitted in this review.

For the two-phase flow in a straight channel with uniform cross sectional area, the total pressure gradient consists of frictional, acceleration, and gravitational contributions [13]. In microchannels, the gravitational contribution is very small compared to the two other factors and can be neglected. Thus the pressure gradient of two-phase flow in microchannels can be regarded as a sum of two terms, frictional and acceleration [15], as:

$$
-\left(\frac{d p}{d z}\right)_{t p}=-\left(\frac{d p}{d z}\right)_{t p, f r}-\left(\frac{d p}{d z}\right)_{t p, a c}
$$

Where subscripts tp, fr and ac represent two-phase property, friction, and acceleration, respectively. The main objectives of those experiments are to obtain empirical correlations of the homogeneous flow model $(f p)$ and the separated flow model (Q. Bowers and Mudawar performed experimental and analytical studies on boiling flow with R-113 in micro- and mini-channels with the hydraulic diameter ranging from $510 \mathrm{pm}$ to $2.54 \mathrm{~mm}$ 
[34]. The homogeneous flow model with $\mathrm{f} p=0.003$, as recommended by Collier, is employed and shows good agreement with experimental data for their microchannel heat sinks (within $30 \%$ of standard deviation) $[17,21]$. Stanley et al. conducted adiabatic two-phase flow experiments using mixed inert gases and liquid water in rectangular channels with hydraulic diameters ranging 56-256 pm [19]. Mishima and Hibiki developed correlation with Z)/, = 1-4 mm [22]. To account for the channel size effect, they modified the expression of $\mathrm{C}$, incorporating the channel hydraulic diameter as:

$$
-\phi_{l}^{1}=1+\frac{C}{X_{v v}}+\frac{1}{X_{v v}^{2}} \text { and } C=21\left[1-\exp \left(-319 D_{h}\right)\right]
$$

Lee and Lee studied same and proposed a various combination of laminar-turbulent flows for each phase and incorporated the liquid phase-alone Reynolds number to include the effects of the channel size and the flow rate $[23,27,34]$. They conducted additional flow boiling experiments with R-113 to further validate their pressure drop model. Qu and Mudawar performed boiling experiments with water in microchannels of $231 \mathrm{pm} \times 713 \mathrm{pm}$. They modified the correlation term to account for the effect of both channel size and mass velocity [26] as follows:

$$
C=21\left[1-\exp \left(-319 D_{h}\right)\right](0.00418 G+0.0613)
$$

They also proposed an annular flow model with empirical droplet entrainment correlation to correlate their data.

\section{Evaporation Models}

\subsection{Slug-annular Flow Model}

A new steady-state slug-annular flow model is developed by $\mathrm{Qu}$ to characterize the heat transfer and pressure drop in microchannels [26]. Figure 2 illustrates the basic geometry of the slug-annular flow in the heated microchannels with a rectangular cross-section presented by $\mathrm{Qu}[26]$. In the two-phase flow regime, there exists a liquid film on the wall while the vapor and liquid slugs flow in the central core $[33,35]$. In this figure, $L_{P}$ is the length of a pair of slugs while $L_{v}$ and $L_{i}$ are the lengths of vapor and liquid bubbles, respectively. The length of the pair as well as the length of the vapor slug increases in the flow direction, due to the applied heat flux. The period of the slug flow is $t_{p}$, in addition $\mathrm{U}$ represents the residence time of vapor and liquid slug at local point, respectively. In the present model, three components exist.
1. Gas slug in the core (moving at the core velocity)

2. Liquid slug in the core (moving at the core velocity)

3. Liquid film on the wall (moving at the film velocity)

Geometry of a slug-annular flow and homogeneous core approximation is shown in Figure 2.

The slug flow is unsteady and oscillating but a steady-state model can be derived in terms of its periodic characteristics. The liquid and vapor slugs may have a secondary fluid motion due to disturbances caused by each other upon initiation of the bubble [11]. This effect is neglected assuming that its effects on pressure and temperature fields are negligible due to the fact that pressure and temperature are governed by the flow in the main stream direction [33]. However, this effect is neglected here since the previous experimental studies on the flow patterns in microchannels show that the vapor-liquid interface is very smooth.

\subsection{Homogeneous Model-slug Flow without Liquid Film}

Another approach for calculating the local pressure of the flow used by Chen is the homogeneous flow model [22]. Critical to the homogeneous flow assumption is that the model assigns identical velocities for the liquid and vapor phases, neglecting the slowly moving liquid film, which dramatically simplifies the mathematical treatment $[2$, 34]. Figure 3 represents this model. Since, in the slug flow model, liquid and vapor slugs move at the same velocity in the core, the homogeneous flow model is the simplest case of the present slug flow model when there is negligible liquid film on the wall. The pressure gradient along the channel is represented as:

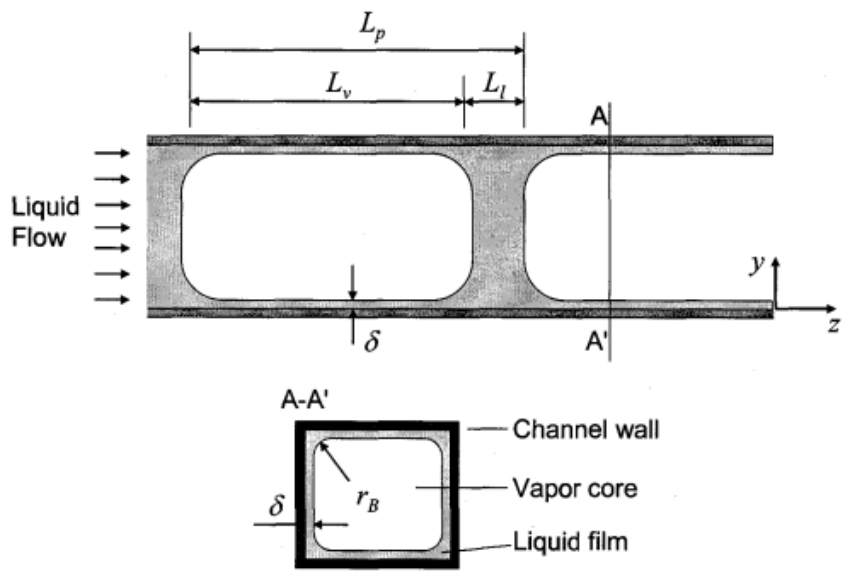

Figure 2. Physical geometry of a slug flow model. 


$$
-\frac{d P}{d z}=\frac{d}{d z}\left(\rho_{t p} U^{2}\right)-f_{t p} \frac{2 G^{2}}{\rho_{t p} D_{h}}
$$

where, the first term represents the acceleration contribution and second term accounts frictional pressure drop. The mass flux of the flow is $G$ and the channel has a uniform cross sectional area along its entire length with a hydraulic diameter of $D_{h}$. The homogeneous two-phase velocity is represented as $\mathrm{U}$. The homogeneous density of the liquid-vapor mixture, $\rho_{t p}$ is evaluated using

$$
\frac{1}{\rho_{t p}}=\frac{(1-x)}{\rho_{1}}+\frac{x}{\rho_{v}}
$$

where, the densities of the liquid and vapor phases are denoted by $\rho_{v}$ and $\rho_{i}$ respectively. To solve the governing set of equations 10 and 11, two complementary relations are required. The most important property characterizing the two-phase microchannel flow is the local fluid pressure. There are several correlations available for heat transfer coefficients and friction factors in two- phase flows [20]. To predict the thermal characteristics of the two-phase flow in microchannels, a proper combination of the correlations and heat transfer coefficients are required since a large change in property is expected due to the significant pressure drop and heat transfer rates in the two-phase region $[22,28]$.

\section{Conclusion}

The boiling flow regimes in microchannels were categorized as the confined bubble, slug flow, and annular flow depending mainly on the length of the bubble. Based on

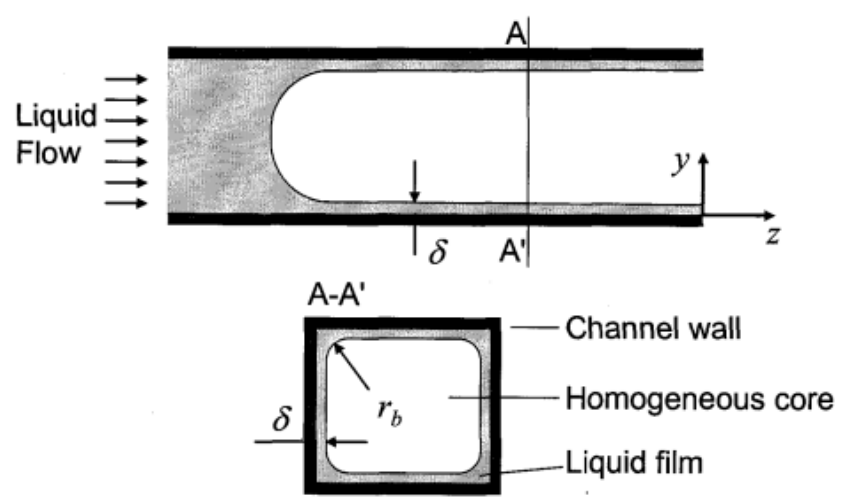

Figure 3. Slug-annular flow model with a homogeneous core [Homogeneous Model]. the dominant flow regimes in microchannels, a steady slug-annular flow model is proposed to predict the pressure drop and heat transfer characteristics. As a correlation method, the homogeneous flow model with empirical correlations was applied to the boiling flow in microchannels. In addition with this, we can also conclude this review in a way that boiling flow in microchannel is an effective way to eliminate heat from the heat sinks and a lot of models are present and proposed by many researchers in past varied on the basis of applications from where it was used.

\section{Acknowledgement}

This work was supported by Universiti Teknikal Malaysia Melaka (UTeM) and Thi-Qar University (Iraq ).

\section{References}

1. Edel Z J, and Mukherjee A (2011). Experimental investigation of vapor bubble growth during flow boiling in a microchannel, International Journal of Multiphase Flow, vol 37(10), 1257-1265.

2. Awad M M (2013). Comments on "boiling heat transfer in rectangular microchannels with reentrant cavities", International Journal of Heat and Mass Transfer, vol 62, 541-542.

3. Shkarah A J, Sulaiman M Y B et al, (2013). Two-phase flow in micro-channel heat sink review paper, The International Review of Mechanical Engineering (IREME), vol 7(1), 231-237.

4. Agostini B et al. (2008). High heat flux flow boiling in silicon multi-microchannels-Part I: heat transfer characteristics of refrigerant R236fa, International Journal of Heat and Mass Transfer, vol 51(21-22), 5400-5414.

5. Shkarah A J, Sulaiman M Y b et al. (2012). Numerical analysis of two phase flow heat transfer in rectangular micro channel heat sink, Archives Des Sciences, vol 65(7), 602-613.

6. Barber $J$ et al. (2009). Hydrodynamics and heat transfer during flow boiling instabilities in a single microchannel, Applied Thermal Engineering, vol 29(7), 1299-1308.

7. Huh C, and Kim M H (2006). An experimental investigation of flow boiling in an asymmetrically heated rectangular microchannel, Experimental Thermal and Fluid Science, vol 30(8), 775-784.

8. Harirchian T, and Garimella S V (2010). A comprehensive flow regime map for microchannel flow boiling with quantitative transition criteria, International Journal of Heat and Mass Transfer, vol 53(13-14), 2694-2702. 
9. Harirchian T, and Garimella S V (2012). Flow regime-based modeling of heat transfer and pressure drop in microchannel flow boiling, International Journal of Heat and Mass Transfer, vol 55(4), 1246-1260.

10. Haynes B S, and Fletcher D F (2003). Subcooled flow boiling heat transfer in narrow passages, International Journal of Heat and Mass Transfer, vol 46(19), 3673-3682.

11. Hsieh S S, and Lin C Y (2012). Correlation of critical heat flux and two-phase friction factor for subcooled convective boiling in structured surface microchannels, International Journal of Heat and Mass Transfer, vol 55(1-3), 32-42.

12. Huh C, Kim J et al. (2007). Flow pattern transition instability during flow boiling in a single microchannel, International Journal of Heat and Mass Transfer, vol 50(5-6) 1049-1060.

13. Sobierska E et al. (2006). Experimental results of flow boiling of water in a vertical microchannel, Experimental Thermal and Fluid Science, vol 31(2), 111-119.

14. Wu H Y, and Cheng P (2003). Liquid/two-phase/vapor alternating flow during boiling in microchannels at high heat flux, International Communications in Heat and Mass Transfer, vol 30(3), 295-302.

15. Kandlikar S G (2010). Scale effects on flow boiling heat transfer in microchannels: a fundamental perspective, International Journal of Thermal Sciences, vol 49(7), 10731085.

16. Sarangi R K, Bhattacharya A et al. (2009). Numerical modelling of boiling heat transfer in microchannels, Applied Thermal Engineering, vol 29(2-3), 300-309.

17. Barber J et al (2010). Bubble confinement in flow boiling of FC-72 in a "rectangular" microchannel of high aspect ratio, Experimental Thermal and Fluid Science, vol 34(8), 1375-1388.

18. Choi $\mathrm{C}$ et al (2011). Flow boiling behaviors in hydrophilic and hydrophobic microchannels, Experimental Thermal and Fluid Science, vol 35(5), 816-824.

19. Kandlikar S G (2006). Nucleation characteristics and stability considerations during flow boiling in microchannels, Experimental Thermal and Fluid Science, vol 30(5), 441-447.

20. Kuo C J, and Peles Y (2009). Pressure effects on flow boiling instabilities in parallel microchannels, International Journal of Heat and Mass Transfer, vol 52(1-2), 271-280.

21. Wang G, Cheng $P$ et al. (2008). Effects of inlet/outlet configurations on flow boiling instability in parallel microchannels, International Journal of Heat and Mass Transfer, vol 51(9-10), 2267-2281.

22. Chen T, and Garimella S V (2011). Local heat transfer distribution and effect of instabilities during flow boiling in a silicon microchannel heat sink, International Journal of Heat and Mass Transfer, vol 54(15-16), 3179-3190.
23. Lee P S, and Garimella S V (2008). Saturated flow boiling heat transfer and pressure drop in silicon microchannel arrays, International Journal of Heat and Mass Transfer, vol 51(3-4), 789-806.

24. Liu Y, Fletcher D F et al. (2013). On the importance of upstream compressibility in microchannel boiling heat transfer, International Journal of Heat and Mass Transfer, vol 58(1-2), 503-512.

25. Wu H Y, and Cheng P (2003). Visualization and measurements of periodic boiling in silicon microchannels, International Journal of Heat and Mass Transfer, vol 46(14), 2603-2614.

26. Qu W et al. (2006). Experimental and computational investigation of flow development and pressure drop in a rectangular micro-channel, Journal of Electronic Packaging, vol 128(1), 1-9.

27. Lee W, Son G et al. (2012). Direct numerical simulation of flow boiling in a finned microchannel, International Communications in Heat and Mass Transfer, vol 39(9), 1460-1466.

28. Celata G P et al. (2010). Heat transfer characteristics of flow boiling in a single horizontal microchannel, International Journal of Thermal Sciences, vol 49(7), 1086-1094.

29. Lee P C, Tseng F G et al. (2004). Bubble dynamics in microchannels. Part I: single microchannel, International Journal of Heat and Mass Transfer, vol 47(25), 5575-5589.

30. Li H Y, Tseng F G et al. (2004). Bubble dynamics in microchannels. Part II: two parallel microchannels, International Journal of Heat and Mass Transfer, vol 47(25), 5591-5601.

31. Kuo C J, and Peles Y (2007). Local measurement of flow boiling in structured surface microchannels, International Journal of Heat and Mass Transfer, vol 50(23-24), 4513-4526.

32. Brutin D, Ajaev V S et al. (2013). Pressure drop and void fraction during flow boiling in rectangular minichannels in weightlessness, Applied Thermal Engineering, vol 51(1-2), 1317-1327.

33. Mosyak A, Rodes L et al. (2012). Boiling incipience in parallel micro-channels with low mass flux subcooled water flow, International Journal of Multiphase Flow, vol 47, 150159.

34. Bertsch S S, Groll E A et al. (2008). Refrigerant flow boiling heat transfer in parallel microchannels as a function of local vapor quality, International Journal of Heat and Mass Transfer, vol 51(19-20), 4775-4787.

35. Mukherjee A, and Kandlikar S G (2009). The effect of inlet constriction on bubble growth during flow boiling in microchannels, International Journal of Heat and Mass Transfer, vol 52(21-22), 5204-5212. 